\title{
Cooperative Media Streaming Using Adaptive Network Compression
}

\author{
Janus Heide, Jesper H. Sørensen, Rasmus Krigslund, \\ Petar Popovski and Torben Larsen \\ Department of Electronic Systems \\ Aalborg University, Denmark \\ $\{$ speje, champz, raskri, petarp, tl $\} @$ es.aau.dk
}

\author{
Jacob Chakareski \\ Layered Media Inc. \\ Hackensack, USA \\ io@jakov.org
}

\begin{abstract}
Media content distribution constitutes a growing share of the services on the Internet. Two distinct distribution approaches used today are Layered Coding (LC) and Multiple Description Coding (MDC). Current wireless connection technologies, e.g. Wimax, have properties which make them unsuitable for media distribution using traditional approaches. In particular, the asymmetric relationship between the uplink and the downlink bandwidth makes the cooperative distribution difficult. A promising concept, termed MDC with Conditional Compression (MDC-CC), has been proposed [11], which essentially acts as an adaptive hybrid between $L C$ and MDC. In order to facilitate the use of $M D C-C C$, a new overlay network approach is proposed, using tree of meshes. A control system for managing description distribution and compression in a small mesh is implemented in the discrete event simulator NS-2. The two traditional approaches, MDC and $L C$, are used as references for the performance evaluation of the proposed scheme. The system is simulated in a heterogeneous network environment, where packet errors are introduced. Moreover, a test is performed at different network loads. Performance gain is shown over both $L C$ and MDC.
\end{abstract}

\section{Introduction}

Distribution of media is a rapidly increasing part of the total Internet traffic. In general, services involving video distribution have very high bandwidth requirements. The tendency is that more media services are distributed via the Internet and, in the near future, via wireless connection technologies e.g Wimax. In such networks, the last hop offers low reliability and the uplink and downlink are highly asymmetric. This results in a generally low and possibly varying QoS $[16,1]$.

Cooperative distribution is one way to overcome the challenge of high bandwidth requirements. In a cooperative approach, users that request the same data at the same time receive disjoint parts of the requested data and cooperatively distribute it among themselves. This reduces the cost of distribution for the provider because the bandwidth consumption of the source is decreased. The available upload capacity is scarce and should be used more efficiently. If nodes primarily cooperate with other nodes to which they have a high connection quality, the amount of late and erroneous received data can be reduced. The nodes can also maximize utilization by exploiting knowledge of the properties of the connections to other nodes and what data is available at those nodes. From this, a node can determine what data to distribute to whom. However, the wireless environment creates largely asymmetric link parameters, which poses a great challenge when combined with cooperative distribution.

A number of cooperative distribution systems and overlay networks approaches have been proposed in recent years. Some systems, such as Pastry [2], target file sharing and similar services, others target multicast streaming. An interesting approach is the Bullet system which utilizes the RanSub algorithm to create overlay meshes on top of an overlay tree [6], [5]. A recurring feature in media distribution systems is to increase available bandwidth and system reliability by utilizing an overlay network. Many of these approaches use a combination of distributed tree and mesh overlay networks to achieve scalability [15]. In media streaming, Tree-First approaches are of particular interest, e.g. ACDC, DCMALTP, HMTP, Hostcast, Overcast, TBCP and Yoid [3], [4], [18], [9], [12]. A very recent and particularly relevant work, presented in [10], proposes to construct a distribution tree by grouping nodes into meshes and connecting the supernodes of these meshes in a tree structure.

The goal is to provide the best possible quality, for the media decoded at the receivers. This introduces the need for coding schemes that define how the media is represented in discrete data blocks. The two coding schemes that are used today are Multiple Description Coding (MDC) and Layered Coding (LC). 
MDC [17] encodes a media stream into multiple nonprioritized descriptions. From any description a low quality replica of the original content can be constructed and every additional description will improve the quality. As the descriptions are self-sufficient, they introduce a large amount of redundancy. This makes MDC error resilient but requires a high level of bandwidth which is not always available, especially not over wireless links.

LC [8] encodes the media into prioritized layers, a base layer and a number of enhancement layers. The enhancement layers depends on the base layer which allows the information in the enhancement layers to be compressed. In this way the encoded media requires less bandwidth compared to MDC, but it is also more susceptible to errors and is therefore not suited for an error prone wireless channel.

Thus MDC and LC are schemes suited for different scenarios in terms of available bandwidth and error rate. In [11] MDC with Conditional Compression (MDC-CC) is introduced. MDC-CC is a scheme that is in between MDC and LC and thus attempts to offer a combination of the advantages of MDC and LC. This is achieved by removing parts of the redundant data introduced by MDC only when this data is rendered useless at the receiver, e.g. when another description has been received. By intelligently removing redundancy MDC-CC can be considered to operate in a cross-layer manner.

Consider the following example where a node has requested a media sequence from a source. The source encodes the sequence into two descriptions, $d_{1}$ and $d_{2}$. If the node successfully receives $d_{1}$ the sequence can be decoded at low quality. If $d_{2}$ is also received the sequence can be decoded at full quality. However, $d_{2}$ contains useless redundant data since $d_{1}$ has already been received. By removing the redundant data, the probability of $d_{2}$ being successfully received increases and the load of the system decreases. However decoding the compressed version of $d_{2}$ depends on $d_{1}$, hence the compressed version of $d_{2}$ is useless if $d_{1}$ is not received.

MDC-CC adaptively alternates between MDC and LC by compressing descriptions. This allows MDC-CC to offer the same error resilience as MDC when the number of errors in the network is the primary constraint and as effective compression as LC when bandwidth is the dominating delimiter. Most often both bandwidth limitation and error state in the network influence the distribution. In this case MDC-CC can offer a redundancy level in between MDC and LC and thus possibly outperform both MDC and LC. This means that MDC-CC potentially allows transfer of media at a higher rate compared to MDC and will maintain higher quality on error prone connections compared to LC.

The remainder of the paper is organized as follows. An overview of the system is given in Section 2. Section 3 presents the scheme utilizing the concept of MDC-CC. Sim- ulation results of the proposed scheme are presented in Section 4. The final conclusions are drawn in Section 5.

\section{System Overview}

An overview of the proposed system is given by introducing its building blocks. In order to develop the system, assumptions about the scenario in which the system must work are specified. The nodes in the scenario are connected to the Internet through a wireless connection. This means that the last hop to every node is wireless. The wireless links are assumed to have different characteristics. All nodes are assumed to have a downlink of $2 \mathrm{Mb} / \mathrm{s}$ and the upload bandwidth ranges from $1 \mathrm{Mb} / \mathrm{s}$ for the strongest node to $160 \mathrm{~kb} / \mathrm{s}$ for the weakest node. The asymmetry between the upload and the download is an important constraint when distributing media content.

\subsection{Tree of Meshes}

The proposed system should facilitate effective and robust distribution of media content. We believe that a tree of meshes has these properties and can be constructed in such a way that is particularly suitable for distribution of multiple descriptions.
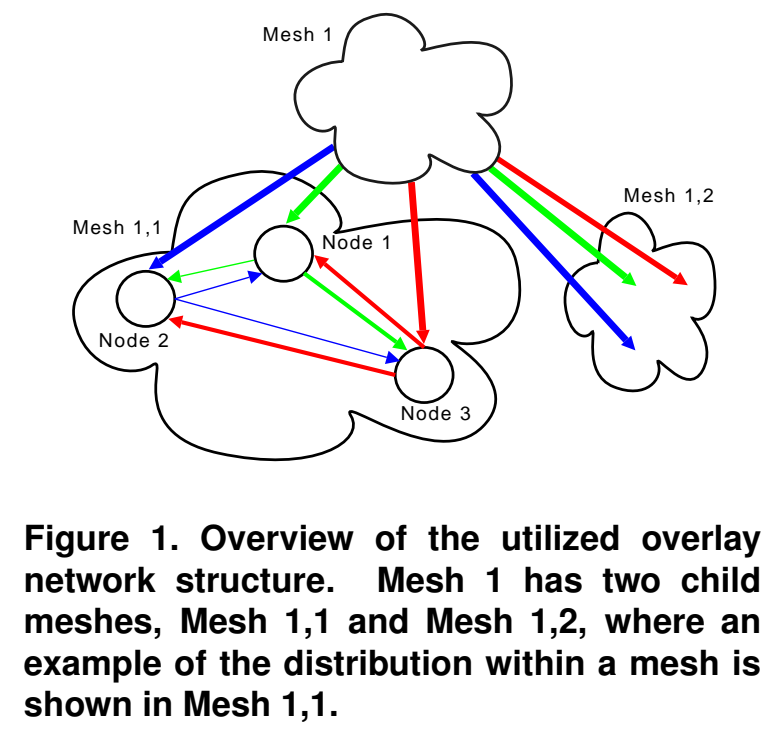

Figure 1. Overview of the utilized overlay network structure. Mesh 1 has two child meshes, Mesh 1,1 and Mesh 1,2, where an example of the distribution within a mesh is shown in Mesh 1,1.

One or more sources transmit different descriptions to the nodes in a mesh, the nodes then forward the description to one or more child meshes. The nodes in the mesh distribute the different descriptions among themselves. In this way the data is only distributed in a peer-to-peer fashion in a small subset of the total nodes. Cooperation between a small subset of nodes instead of all nodes in the network is simpler. Therefore cooperation in a tree of meshes is much 
simpler compared to flat networks or networks where cooperation is done between clusters of nodes.

The overlay network must be constructed in such a way that the latency between the nodes in a mesh is low. In this way the nodes can quickly react to changing network conditions within the mesh and retransmit data without incurring a large delay. Furthermore the tree should be balanced in such a way that meshes with high overall upload capacity are positioned higher up in the tree. However, the construction of the overlay network is outside the scope of this paper, instead the main focus is to develop a scheme for distribution of descriptions within a mesh after they are received from a source. It is assumed that the nodes in a mesh receive their initial description at approximately the same time.

\subsection{Transcoding}

From each Group Of Frame (GOF) descriptions are encoded using an MDC-FEC transcoder [13]. By protecting each layer with FEC, MDC-FEC is able to convert a layered media stream into multiple descriptions. The FEC is encoded using $(n, k)$ Reed Solomon codes, where $k$ is the length of the data and $n$ the total codeword length. In this way the original data can be reconstructed from any $k$ symbols of the $n$ symbol codeword. When the FEC is appended the resulting descriptions are created by including one part from each layer. Figure 2 shows an example of this for four layers and four descriptions.

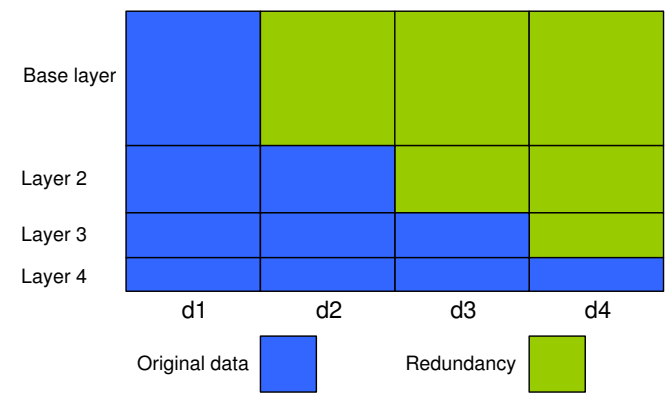

Figure 2. The descriptions are created using the principle of MDC-FEC.

\section{[14]}

The base layer must be decodable if just one description is received and is therefore encoded with the strongest FEC. The strength of the FEC decreases up through the enhancements layers. Therefore the second layer (first enhancement layer) is decodable when any two descriptions are received and the $k$-th layer is decodable when any subset of $k$ descriptions is received.

\section{Scheme Proposal}

The objective of the scheme is to efficiently distribute descriptions between a set of nodes in a small mesh. The key idea is to compress descriptions, distribute these descriptions the same way as for the last GOF and send control messages if too little or too much data was received for the last GOF. Due to the asymmetry of typical wireless connections, if the downlink capacity is fully utilized it is not possible to forward the same amount of data via the uplink. Hence, in such cases, cooperative distribution is only possible if compression is performed.

In the simplest example two nodes receive disjoint descriptions from a source. The nodes exchange a compressed version of the original descriptions, such that the quality of the decoded media is improved at both nodes. This lowers the bandwidth requirement compared to the case where the descriptions are not compressed. This is illustrated in Figure 3.

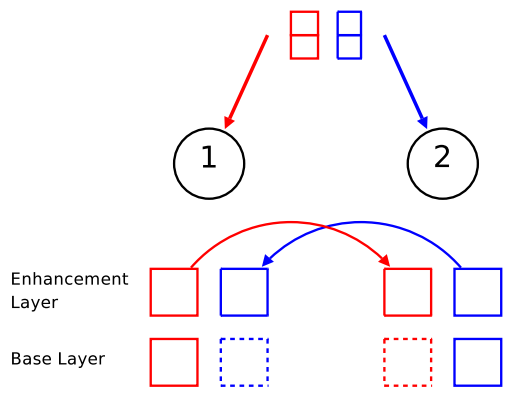

Figure 3. Two nodes receive data from a source and exchange a compressed description in order to efficiently improve the media quality.

Initially, the nodes in the mesh will only receive a description from their parent. In order for the distribution within the mesh to commence, the nodes need to determine who they should send their description to and how much it should be compressed. Figure 4 shows how this should be done through illustrations of the distribution rounds for GOF one to four. In Figure 4(a), the distribution of the first GOF is illustrated. Each of the nodes only receives one description from the source and hence all nodes can only decode the base layer. Then, each node sends a request for an additional description from a random node in the mesh in order to improve the distribution of the media content in the next GOF. This additional description that will be sent to the requesting node can have the base layer removed prior to transmission. That is because the requesting node will receive, with high probability, a complete description from the source in the next GOF from which the node can reconstruct the base layer by itself. In Figure 4(b) each node 
receives a description from the source and one compressed description from the mesh as requested in the previous GOF. This makes all nodes able to decode two layers. Finally, the nodes request a description where two layers (base + one enhancement) are removed in order to facilitate decoding at full quality at each node in the next GOF. In Figure 4(c), the system has converged as all nodes can decode all layers. Hence, no control messages are needed in the following round.

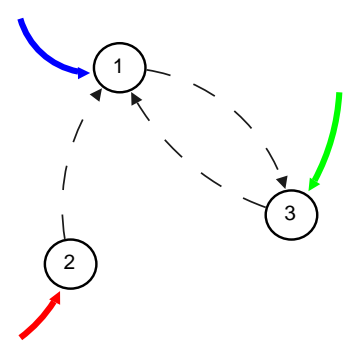

(a) Distribution of GOF

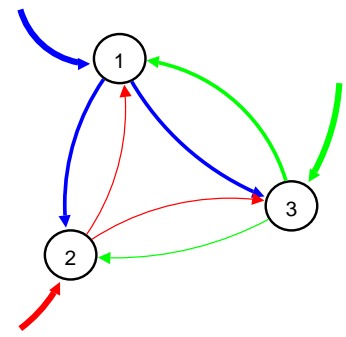

(c) Distribution of GOF 3

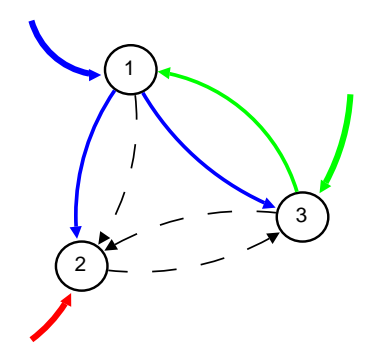

(b) Distribution of GOF

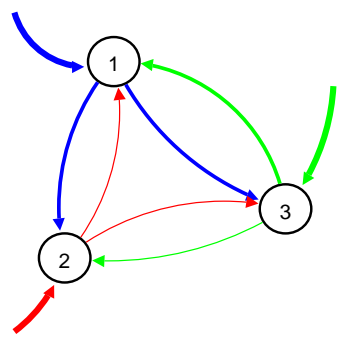

(d) Distribution of GOF 4
Figure 4. The width of the arrows denotes the compression level of the descriptions. A thinner arrow denotes a higher compression level. Dashed lines denote control messages. Different colors denote different descriptions.

To realize this a control system is introduced, which allows each node to request descriptions from all other nodes in the mesh and to specify the level of compression. Upon decoding, if a node detects that insufficient data was received to decode at full quality, it will determine which nodes it can request more data from to improve the situation. It will choose one at random and it will send a control message to this node to decrease the compression level for the next GOF. Similarly, if too much data is received, the node will choose a node at random and increase its com- pression level.

A generalization of the functionality of the scheme is formulated in the following and pseudocode is listed in Listing 1. A small amount of nodes are organized in a mesh. When they receive a description from their parents they forward this to their children. The nodes cooperatively distribute the descriptions among themselves and compress descriptions when possible. This is achieved by having each node send requests for changes in the compression level if the compression was suboptimal in the last distribution round. If an expected description was not received, a node can send an emergency request to one or more nodes in order to recover the lost data.

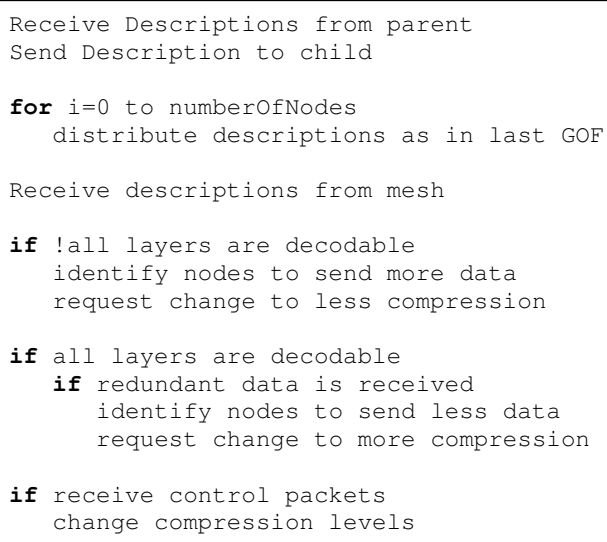

\section{Listing 1. Outline of the steps performed for the distribution of each GOF}

\subsection{Dynamic Redundancy Level}

The control system of the scheme is controlling the level of redundancy in the descriptions that are forwarded by adjusting the level of compression. During the transmissions in which no errors occur in the network, the desired steady state in the control system is full compression of the descriptions, which results in no redundancy. However, when errors do occur in the network, a certain amount of redundancy is required in order to cope with the errors. This is achieved by changing the desired steady state in the control system, and have it converge to another level of compression. In this way the level of redundancy will dynamically adapt to a given network state.

If an error occurs in a system with $n$ descriptions, which is in a state of full compression, the outcome is the loss of $k$ layers, where $k$ is between 1 and $n$. We wish to decrease the impact of such an error by introducing redundancy. In order to adapt to a given network state, it is necessary to collect information about the performance of the network. In this paper we use the following heuristic approach. We 
introduce a vector, $\bar{v}$, which is initialized as $\overline{0}$ and which entries describe the number of errors that have occurred for each description. In particular, when an error occurs in the $j$ 'th description, $\bar{v}(j)$ is incremented by one, and for each description set $\bar{v}$ is multiplied with the scalar $V \epsilon\{0,1\}$. This ensures that recent errors are weighted highest. An error is defined as loss of a requested layer in a description.

The amount of redundancy should be proportional to $\bar{v}(j)$, since a high value indicates a high probability of error. Hence if $\bar{v}$ contains all zeros, no redundancy is needed, and the steady state is set to full compression. In the following example with four descriptions two errors occur.

Initially the system is in a steady state with full compression and the error vector contains all zeros, see Figure 5(a). Hence, no redundancy is added to the descriptions. Two errors occur in description 2 and $\bar{v}(2)$ is incremented accordingly. Therefore, the redundancy level is increased in the descriptions that have higher compression level than $\mathrm{d} 2$, in this case $d 3$ and $d 4$, see figure 5(b). If no further errors occur, $\bar{v}$ will decrease and the desired steady state will return to full compression again.

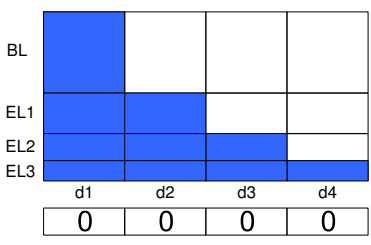

(a) No errors

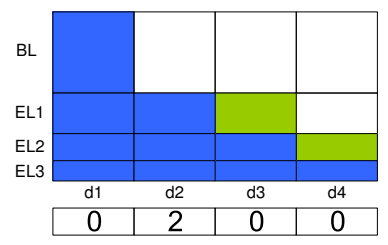

(b) Two errors

\section{Figure 5. The description set when (a) no er- rors have been detected, (b) 2 errors have been detected in description 2.}

This mechanism makes the proposed scheme operate as an adaptive hybrid between LC and MDC. A given network state described by the error vector, $\bar{v}$, yields a corresponding level of redundancy in the system. One extreme is $\bar{v}=\overline{0}$ which leads to no redundancy in the system, which is comparable to LC. Another extreme is when $\bar{v}$ contains only non-zero elements, and the values are high enough to require maximum redundancy in the system, which is comparable to MDC.

\section{Results}

To evaluate the performance of the proposed scheme a test scenario is simulated using the discrete event simulator NS-2 ${ }^{1}$. The proposed scheme is designed to combine ele-

\footnotetext{
${ }^{1}$ http ://nsnam.isi.edu/nsnam/index.php/User_Information
}

ments from MDC and LC and the purpose of this simulation is therefore to show in which situations the proposed scheme yields a performance improvement over the two traditional approaches. Due to the extensive redundancy, MDC is suited for error prone scenarios with a relatively high available bandwidth. LC is suited for scenarios with approximately no errors and limited bandwidth since LC contains a minimum amount of redundancy. It is therefore chosen to simulate the schemes in scenarios where the network parameters are set up to suit MDC and LC.

We simulate a mesh of four nodes connected by UDP connections where the upload capacity in the mesh is distributed heterogeneously using the bandwidths $384 \mathrm{~kb} / \mathrm{s}$, $192 \mathrm{~kb} / \mathrm{s}$ and two nodes with $60 \mathrm{~kb} / \mathrm{s}$. The download capacity is $2 \mathrm{Mb} / \mathrm{s}$ for all nodes and the multiplicative factor $V$ defined in Section 3.1 equals 0.9 .

The simulation uses artificial data to represent a real media stream where each GOF is encoded into four descriptions. This means that we can abstract from any transcoding of data, instead descriptions are a sequence of random data with a size relative to the media size in use. The rate allocation among the layers in each description is as follows; base layer: $50 \%$, enhancement layer 1: $25 \%$, enhancement layer 2: $15 \%$, and enhancement layer 3: $10 \%$. If a real media sequence were used the descriptions could be constructed using MDC-FEC, as described in section 2.2.

In its current state the scheme only allows nodes to distribute the description they received from their parent mesh. In order to obtain minimum distortion a node must receive $n$ parts of the $n^{\text {th }}$ layer, as would be the case if a real media sequence were encoded and distributed. Since artificial data is used, it is not possible to measure the distortion on real media. Instead we estimate the distortion based on the amount of received useful data. The used distortion measure is Mean Squared Error (MSE) and to calculate the distortion the following rate distortion function has been estimated for the applied H.264 codec:

$$
D(R)=e^{4.5792-0.00011028 \cdot R}
$$

Any amount of received data in the simulations can be interpreted as a certain source encoding rate. Hence, the MSE is calculated during the simulations using equation (1).

\subsection{Varying Media Size}

In order to compare the proposed scheme with LC, a scenario with conditions optimized for LC is set up. Since the proposed scheme is designed for meshes with heterogeneous distribution of upload connections, the simulation is performed in such a scenario. The simulation is run using media sizes from $1 \mathrm{kB} / \mathrm{GOF}$ to $25 \mathrm{kB} / \mathrm{GOF}$ in steps of $1 \mathrm{kB}$. 


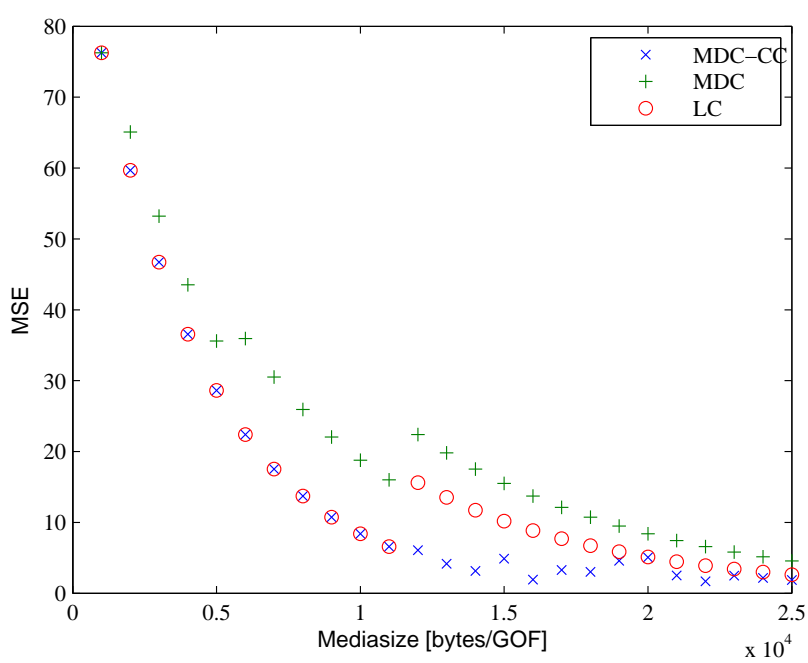

Figure 6. The experienced distortion as a function of media size when the upload capacity is distributed heterogeneously for the proposed scheme, MDC and LC.

The scatter plot in figure 6 shows the distortion experienced using the three schemes during the simulation as a function of the media size. The distortion decreases as the media size increases. Note that the reduction in distortion is due to contributions from both the source and the distribution mesh. At some point no data is received from the mesh, hence there is no cooperation in the mesh and the distortion only depends on data received from the source.

In the first sample all three schemes are able to decode all the layers. In the second and sixth sample MDC loses the third and second enhancement layer, respectively. At a media size of $12 \mathrm{kB} / \mathrm{GOF}, \mathrm{LC}$ is unable to distribute the media and loses all the enhancement layers and MDC loses the remaining enhancement layer. From that point on, MDC and LC rely entirely on the base layer while the proposed scheme is able to deliver all layers.

LC is hardcoded to distribute the load equally among the nodes and MDC-CC performs therefore better than LC because the load on the nodes is dynamic. When the media size reaches $12 \mathrm{kB} / \mathrm{GOF}$, the slowest nodes are overloaded and the quality for LC is significantly degraded. If we observe MDC in this scenario, we see a similar effect, but at smaller media sizes.

\subsection{Varying Packet Error Rate}

The proposed scheme is compared with MDC by simulating a scenario with packet errors in order to make use of the redundant data in MDC. Packet errors are introduced in the system at 30 different rates from $10^{-4}$ to $2 \cdot 10^{-1}$ in logarithmic steps, while the media size is held constant at 5 $\mathrm{kB} / \mathrm{GOF}$.

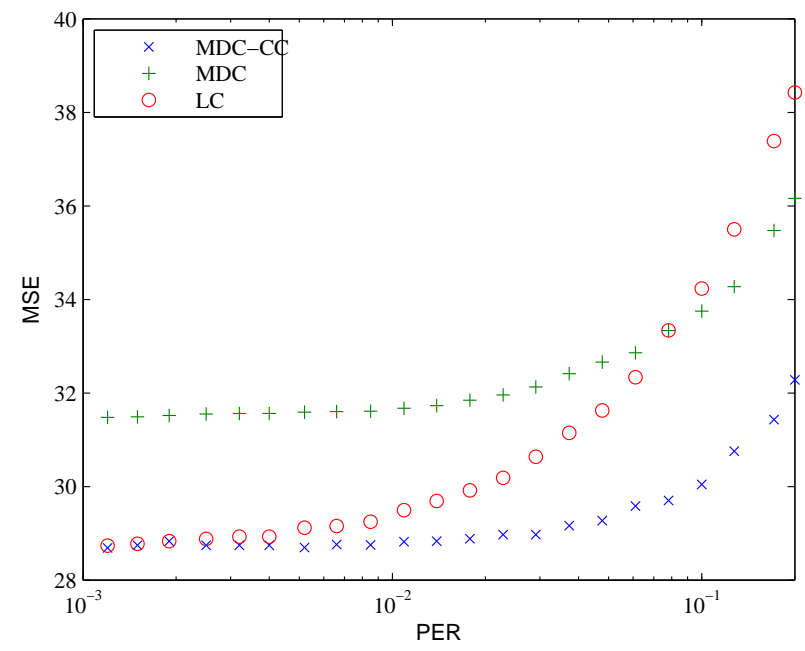

Figure 7. The experienced distortion plotted as a function of the rate of introduced errors in a scenario with heterogeneous distributed upload capacity.

The experienced distortions vs. varying PER are plotted in Figure 7. As the error rate increases, the distortion increases for all three schemes. MDC experiences a high distortion over the entire PER range due to the heterogeneous distribution of upload capacity, which makes the weak nodes unable to distribute the uncompressed descriptions. LC and the proposed scheme distribute maximally compressed descriptions and therefore experience comparable distortions at low PERs. As PER increases, the proposed scheme is able to adapt the level of redundancy in order to cope with the errors. The distortion of LC increases more rapidly and for PER $\geq 10^{-1}$, LC experiences a higher distortion than MDC.

In Figure 7, the slopes of the three graphs increase differently with the PER indicating the impact of the introduced errors on each of the schemes. The slope of LC rises faster than those of MDC and the proposed scheme. This indicates that LC has a lower error resilience than the two other schemes.

\section{Conclusion and Future Work}

A scheme based on the concept of Multiple Description Coding with Conditional Compression (MDC-CC) has been presented. The idea of the scheme is to combine the virtues of MDC and Layered Coding (LC). MDC-CC is particularly suitable for cooperative distribution of the multimedia content in cases where the downlink/uplink capacities of the 
terminal nodes exhibit a high asymmetry (which is the practical case). Performance evaluations in NS-2 using heterogeneously distributed upload capacities show that the proposed scheme performs significantly better than MDC and LC in a scenario with increasing network load and in a scenario with increasing PER. These simulations indicate that the proposed scheme is able to distribute the load between the nodes in the mesh in order to adapt to the varying upload bandwidths, thereby utilizing the available resources more efficiently. Moreover the scheme is able to adaptively adjust the level of redundancy to increase the error resilience on the channels with high error probability.

One avenue of future work can be development of an optimized algorithm for dynamic selection of the redundancy level in the system. Currently, a heuristic approach is used and it might be beneficial to select the employed redundancy based on an estimate of the expected reconstruction distortion at the nodes for each possible redundancy level. With proper network state information these estimates can be made and the redundancy level with the best performance estimate can be selected. Work related to this approach is described in [7].

\section{References}

[1] Anmol Sheth, Sergiu Nedevschi, Rabin Patra, Sonesh Surana, Eric Brewer, Lakshminarayanan Subramanian. Packet Loss Characterization in WiFi-based Long Distance Networks. In IEEE INFOCOM 2007 - 26th IEEE International Conference on Computer Communications, pages 312 - 320, Anchorage, Alaska, USA, 6-12 May 2007. INFOCOM 2007, IEEE.

[2] Antony Rowstron and Peter Druschel. Pastry: Scalable, Decentralized Object Location, and Routing for Large-Scale Peer-to-Peer Systems. In IFIP/ACM International Conference on Distributed Systems Platforms, pages 329-350, Heidelberg, Germany, 12-16 November 2001. Springer-Verlag, Berlin.

[3] S. Banerjee, C. Kommareddy, K. Kar, B. Bhattacharjee, and S. Khuller. Construction of an efficient overlay multicast infrastructure for real-time applications. In INFOCOM 2003. Twenty-Second Annual Joint Conference of the IEEE Computer and Communications Societies. IEEE, volume 2, pages 1521-1531, San Fransisco, CA, USA, 30 march - 3 April 2003.

[4] Beichuan Zhang Sugih Jamin Lixia Zhang. Host Multicast: A Framework for Delivering Multicast to End Users. In Twenty-First Annual Joint Conference of the IEEE Computer and Communications Societies INFOCOM-02, volume 3, pages 1366-1375, New York, NY, USA, 23-27 June 2002. IEEE.

[5] Dejan Kostic, Adolfo Rodriguez, Jeannie Albrecht, Abhijeet Bhirud, and Amin Vahdat. Using Random Subsets to Build Scalable Network Services. In 4th USENIX Symposium on Internet Technologies and Systems. USENIX Association, 26-28 March 2003.
[6] Dejan Kostic, Adolfo Rodriguez, Jeannie Albrecht, and Amin Vahdat. Bullet High Bandwidth Data Dissemination Using an Overlay Mesh. In ACM SIGOPS Operating Systems Review, volume 37, pages 282-297. ACM New York, NY, USA, December 2003.

[7] Dilip Krishnaswamy, Hsien-Po Shiang, John Vicente, W. Steven Conner, Sanjay Rungta, Winson Chan, Kai Miao. A Cross-Layer Cross-Overlay Architecture for Proactive Adaptive Processing in Mesh Networks. In Proceedings of the 2nd IEEE workshop on Wireless Mesh Networks, pages 74-82, Reston, VA, USA, 25 September 2006. IEEE.

[8] Hayder Radha, Yingwei Chen, Kavitha Parthasarathy, Robert Cohen. Scalable Internet video using MPEG-4. In Signal Processing: Image Communication, volume 15, pages 95-126, Philips Research, 345 Scarborough Rd, Briarcliw Manor, New York, 10510, USA, 4 October 1999. Elsevier Science B.V.

[9] John Jannotti, David K. Gifford, Kirk L. Johnson, M. Frans Kaashoek and James W. O'Toole, Jr. Overcast: Reliable Multicasting with an Overlay Network. In Proceedings of the 4th conference on Symposium on Operating System Design \& Implementation - Volume 4, page 14, San Diego, CA, USA, 23-25 October 2000. USENIX Association.

[10] F. A. López-Fuentes and E. Steinbach. Hierarchical collaborative multicast. In The 15th international conference on Multimedia 07, pages 763-766, Augsburg, Germany, 23-29 September 2007. ACM, New York, NY, USA.

[11] M. H. Larsen, P. Popovski, and S. V. Andersen. Cooperative Communication with Multiple Description Coding. In Cooperation in Wireless Networks: Principles and Applications, pages 515-545. Springer, 2006.

[12] Paul Francis. Yoid: Extending the Internet Multicast Architecture. ISI, 2000. Available online: http://www.aciri.org/yoid/docs/index.html.

[13] Rohit Puri, Kannan Ramachandran. Multiple Description Source Coding using Forward Error Correction Codes. In Conference Record of the Thirty-Third Asilomar Conference on Signals Systems and Computers (Cat No CH37020) ACSSC-99, volume 1, pages 342-346, Pacific Grove, CA, USA, 24-27 October 1999. UC, Berkeley.

[14] Rohit Puri, Kannan Ramachandran, K.W. Lee, V. Bharghavan. Forward error correction (FEC) codes based multiple description coding for internet video streaming and multicast. volume 8. Elsevier Science, 2001.

[15] Tan Su-Wei, Gill Waters and John Crawford. A Survey and Performance Evaluation of Scalable Tree-based Application Layer Multicast Protocols. In Technical Report, No. 9-03. University of Kent, Canterbury,, 2003.

[16] Vern Paxson. End-to-End Routing Behavior in the Internet. In ACM SIGCOMM Computer Communication Review, volume 36, pages 41-56. ACM New York, NY, USA, October 2006.

[17] Vivek K Goyal. Multiple Description Coding: Compression Meets the Network. IEEE Signal Processing Magazine, 18:74 - 93, 2001.

[18] Zhi Li and Prasant Mohapatra. HostCast: A New Overlay Multicast Protocol. In IEEE International Conference on Communications 2003 ICC 03 ICC-03, volume 3, pages 702-706, Anchorage, Alaska, USA, 11-15 May 2003. IEEE. 